Check for updates

Cite this: Mater. Horiz., 2020,

7, 1872

Received 29th February 2020

Accepted 29th April 2020

DOI: $10.1039 / \mathrm{d} 0 \mathrm{mh} 00361 \mathrm{a}$

rsc.li/materials-horizons

\section{Stretchable, self-healing and tissue-adhesive zwitterionic hydrogels as strain sensors for wireless monitoring of organ motions $\dagger$}

\author{
Xinjie Pei, ${ }^{\text {abc }}$ Hua Zhang, ${ }^{b}$ Yang Zhou, ${ }^{b}$ Linjie Zhou ${ }^{a}$ and Jun Fu (D) *a
}

\begin{abstract}
Skin-inspired sensors have great potential applications in wearable and implantable devices to monitor human motions. Robust tissue adhesion, fatigue resistance, and biocompatibility are desired for in situ signal capture and wireless transmission. Inspired by the mussel and zwitterionic adhesion mechanisms, we prepared novel stretchable, self-healing and polydopamine zwitterionic nanocomposite hydrogels that provide reversible and robust adhesion to tissues with a strength up to $19.4 \mathrm{kPa}$ and a strain sensitivity of 4.3. The hydrogel sensors robustly adhere to organs like the heart, liver, and lungs to capture signals for remote monitoring and diagnostics through wireless transmission.
\end{abstract}

\section{Introduction}

Implantable biomedical devices have been widely used to monitor the physiological status of patients and treat diseases, including pace-makers, implantable cardioverter defibrillators (ICDs) and retinal visual prostheses. ${ }^{1-4}$ Recently, wearable and implantable biosensors and devices have shown new impacts on the body sensor network (BSN), a fusion of sensors, Internet of Things (IoT) technology, ${ }^{5}$ and wireless communication technology, for real time physiological monitoring of the elderly and patients with chronic diseases. Miniaturized low-power biosensors and a wireless network are the basic requirements for this purpose. ${ }^{1}$ There are still inherent gaps between artificial devices and biological tissues. Most current implantable electronic devices are fixed in the patient by suturing, which usually involves a mismatch in biocompatibility and mechanical properties, and damage to host tissues. ${ }^{6-8}$ Hydrogels comprised of crosslinked hydrophilic polymers are similar to the extracellular matrix and are thus ideal candidate materials for implantable

\footnotetext{
${ }^{a}$ School of Materials Science \& Engineering, Sun Yat-sen University, Guangzhou 510275, P. R. China.E-mail: fujun8@mail.sysu.edu.cn

${ }^{b}$ Polymers and Composites Division, Ningbo Institute of Materials Technology and Engineering, Chinese Academy of Sciences, Ningbo 315201, P. R. China

${ }^{c}$ University of Chinese Academy of Sciences, Beijing 101408, P. R. China

$\dagger$ Electronic supplementary information (ESI) available. See DOI: 10.1039/d0mh00361a
}

\begin{abstract}
New concepts
Skin-inspired pressure and strain sensors have great potential applications in wearable and implantable devices to monitor human motions. Ion conductive hydrogels are advantageous as flexible sensors for implantable devices. Wireless signal transmission is needed for remote monitoring of target organs. Here, we demonstrate novel tissue adhesive ion conductive hydrogels as sensitive strain sensors. Inspired by mussel adhesion to diverse substrates, polyzwitterionic polymers are crosslinked by dopamine-modified clay nanosheets to prepare hydrogels with outstanding stretchability, self-healing, fast recovery, and fatigue resistance. The hydrogels are sensitive to stretching with a gauge factor of 4.3. The synergistic contributions from the zwitterionic monomers and catechol groups give robust adhesion to various substrates, including the skin, liver, lungs, and heart in air and under water. Different from most other flexible elastomer or hydrogel sensors reported in the literature, our current hydrogels are able to establish robust and conformal adhesion to target organs, which survives vigorous vibrations. The hydrogel sensors adhered to skin or organs not only seal the wound, but also provide high sensitivity to subtle vibrations and motions. We demonstrate wireless transmission of signals captured by the hydrogel sensors. It opens a novel avenue for implantable wireless devices free of suturing.
\end{abstract}

devices. Biomimetic structures, robust adhesion and conformal contact of sensors with target tissues or organs are desired to efficiently detect motions of target organs for conversion into electrical signals.

Mussel-inspired hydrogels are adhesive to diverse substrates. ${ }^{9-11}$ Regardless of the surface roughness and environmental humidity, mussels adhere firmly to almost all surfaces through noncovalent interactions and covalent bonding with substrates. ${ }^{10}$ Elastin-like polypeptides with tyrosine residues and 3,4-dihydroxyphenylalanine (DOPA) molecules showed underwater adhesion with a strength up to $0.24 \mathrm{MPa}$, but they are not reusable. ${ }^{12}$ Clay-initiated polydopamine-polyacrylamide (PDA-PAM) composite hydrogels provide repeatable and long-lasting adhesion to hydrogels, with an adhesion strength up to $28.5 \mathrm{kPa}$ on pig skin. ${ }^{13}$ Therein, the free catechol groups in the PDA-intercalated clay nanosheets enable re-adhesion to pig skins. However, most musselinspired hydrogels are stretchable and self-healable, but weak. 
The adhesion performance may be compromised for hydrogels with high strength and modulus.

Stability and reliability are critical for practical applications as implantable sensors, which demand conductive, highly stretchable, durable and tissue-adhesive materials. ${ }^{14-16}$ Many electro-conductive flexible devices have been manufactured by incorporating metal nanomaterials, ${ }^{17-19}$ carbon nanomaterials, ${ }^{20,21}$ and conductive polymers ${ }^{22,23}$ into elastomers or hydrogels. Ion conduction is ubiquitous and critical for biological signal transmission from neurons to the brain and muscles. $^{24}$ Ion conductive polyelectrolyte hydrogels ${ }^{25}$ are biomimetic and adaptable to biotissues, and have shown high conductivity and sensitivity. For example, a plant-inspired zwitterionic hydrogel composited and crosslinked with cellulose nanocrystals $^{26}$ serves as a stretchable strain sensor to monitor human joint activity and talking, with a strain sensitivity (gauge factor) of 1.3 at $100 \%$ strain. Supramolecular sodium alginate (SA) nanofibrillar double network hydrogels showed a strain sensitivity of $\sim 2.0$ below $200 \%$ strain and a wide strain window $(0.3-1800 \%){ }^{27}$ Inspired by the aligned structures in muscles, $\mathrm{Hu}$ et $a .^{28}$ prepared conductive wood hydrogels by strongly bonding and cross-linking cellulose nanofibers (CNFs) and polyacrylamide in wood. The negatively charged aligned CNFs give an ion conductivity up to $5 \times 10^{-4} \mathrm{~S} \mathrm{~m}^{-1}$.

In order to monitor the target organ in real time with minimal loss in signal transmission, excellent adhesion on the tissue surface is needed for implanted hydrogel sensors. Park et al. incorporated PDA and tannic acid into hydrogel networks to achieve adhesion to fingers. ${ }^{29}$ Wan et al. composited PVA hydrogels with functionalized single-wall carbon nanotubes and polydopamine to achieve an adhesion strength of $5 \mathrm{kPa}$ to pigskin. ${ }^{30}$ Gao et al. devised a hydrogel bilayer device comprised of a robust conductive layer and an adhesive layer. Therein, the adhesive layer provided an adhesion of $130.9 \mathrm{~N} \mathrm{~m}^{-1}$ to pig skin, while the ion conductive layer served as a strain sensor to monitor human motions. ${ }^{31}$ Zwitterionic hydrogels are able to adhere to diverse substrates through ion-dipole or dipole-dipole interactions, ${ }^{32-34}$ but with low adhesion strength ( $8 \mathrm{kPa})$. PVA/PSBMA (polyvinyl alcohol/poly[2-(methacryloyloxy)ethyl] dimethyl-(3-sulfopropyl) ammonium hydroxide) hydrogels show repeatable adhesion to substrates and high linear strain sensitivity. ${ }^{35}$ But its adhesion strength on pig skin was only $5.22 \mathrm{kPa}$, lower than that of fibrin glue on pig skin $\left(16.5 \mathrm{kPa}^{36}\right)$. To achieve robust hydrogel adhesion to biotissues, we proposed a co-adhesive mechanism combining mussel-like and zwitterionic adhesion mechanisms on the surface of target tissues or organs.

In this work, we demonstrate novel tissue-adhesive ion conductive hydrogel sensors for remote monitoring of organ motions through wireless transmission. Zwitterionic hydrogels composited with dopamine-modified clay nanosheets (Fig. 1a) were prepared. The dopamine monomers could adsorb on clay nanosheets and some may intercalate into the layers. Upon oxidative polymerization, most of the monomers are converted into PDA, whereas some may remain catechol groups, ${ }^{37}$ as schematically illustrated in Fig. 1a. The free catechol groups may allow for the formation of hydrogen bonding, Michael addition, and Schiff base reactions at the hydrogel-tissue interface, which work synergistically with zwitterionic dipole-dipole interactions to provide robust and reusable adhesion to biotissues (Fig. 1b). ${ }^{38-40}$ The nanocomposite and zwitterionic nature gives not only high stretchability, but also self-healing to the ion conductive hydrogels. The hydrogels show an ion conductivity of $0.0215 \mathrm{~S} \mathrm{~m}^{-1}$ and a high strain sensitivity (gauge factor GF $=4.3,0-670 \%$ strain). (a)

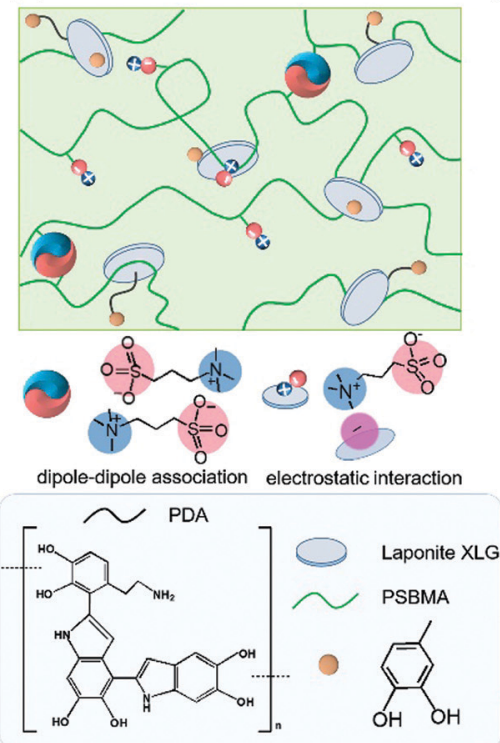

(b)

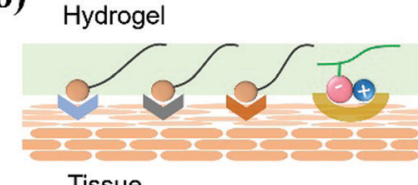

Tissue
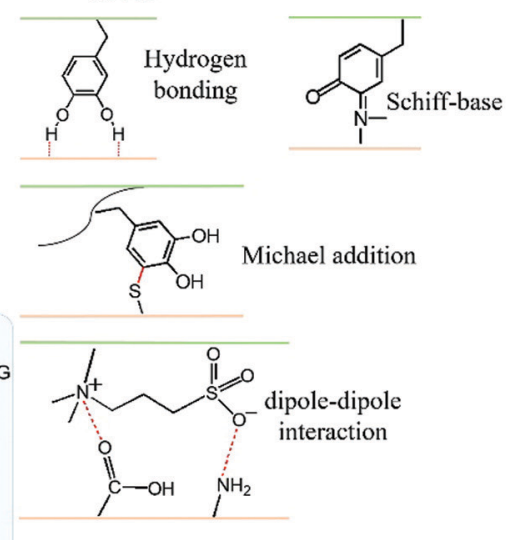

(c)

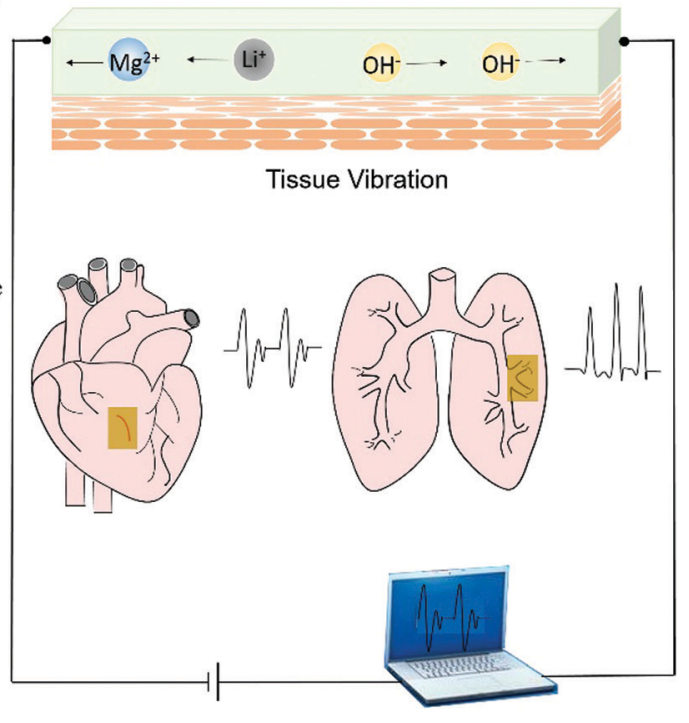

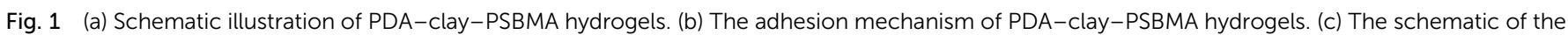
hydrogel sensor for monitoring tissue vibration. 
Robust adhesion of the hydrogels to biotissues (adhesion strength $19.4 \mathrm{kPa}$ ) establishes conformal and compliant contact between the hydrogels and target organs. The dynamic vibration of organs is monitored by the hydrogel sensors, generating electric signals to the computer through wireless transmission (Fig. 1c). The hydrogels are promising for wearable and implantable tissue adhesion sensors in vivo.

\section{Results and discussion}

Synthesis, microstructures, and mechanical properties of the hydrogels

The hydrogels were prepared by one-pot free radical polymerization of SBMA with the presence of dopamine-modified clay and MBAA with a molar ratio to SBMA of $3 \times 10^{-4}$. The amino groups of PDA may form an electrostatic interaction with clay nanosheets, ${ }^{41}$ and phenolic hydroxyl groups may chelate with ions on the clay surfaces, thus anchoring to the clay nanosheets. ${ }^{42,43}$ On the other hand, due to the dissolution of ions from clay, the clay nanosheets may become alkaline, which is conducive to self-polymerization of DA into PDA. Moreover, the SBMA monomers can adsorb onto the clay nanosheets through electrostatic interactions with the negative charges on the surfaces and positive charges on the edges. ${ }^{44}$ Free radical polymerization yielded hydrogels crosslinked by physical adsorption and sparse chemical crosslinking. Meanwhile, the zwitterionic moieties can form dipole-dipole association (Fig. 1a) and thus provide additional physical cross-linking to the network. ${ }^{19}$ During this procedure, the variations in clay spacing are investigated by using wide angle X-ray diffraction (WAXD) and transmission electron microscopy (TEM). The presence of PDA and SBMA decreased the Bragg angles and thus increased the spacings of the clay. No diffraction peaks were observed for the PDA-PSBMAclay hydrogels (Fig. 2a). These findings are consistent with TEM observations of microtomed PDA-PSBMA-clay hydrogels (Fig. 2b), which suggest complete exfoliation of clay nanosheets in the hydrogels.

The PDA-clay-PSBMA hydrogels exhibited outstanding stretchability and toughness. The presence of PDA generated microfibers in the hydrogels (Fig. S1, ESI $\dagger$ ), which may favour high stretchability and toughness. Fig. 2c shows representative tensile stress-strain curves of hydrogels with different clay contents. As the clay content was increased from 0.01 to $0.03 \mathrm{~g} \mathrm{~mL}^{-1}$, the tensile strength increased monotonically from 54 to $90 \mathrm{kPa}$, while the fracture strain remained around 700$900 \%$. On the other hand, the nanocomposite hydrogels showed a compression strength from 3.8 to $6.2 \mathrm{MPa}$ with increasing clay content (Fig. 2e). The high clay contents significantly enhanced the mechanical strength and toughness of the gels. Cyclic loading-unloading tensile tests to $400 \%$ strain resulted in large hysteresis loops (Fig. 2d). The loop area indicates the energy dissipation during loading. For the second and subsequent cycles, the loop areas decreased to $58.5 \%$ of the original value. Meanwhile, the loading-unloading curves overlapped each other, indicating a rapid recovery of the hydrogel during cyclic loadings.
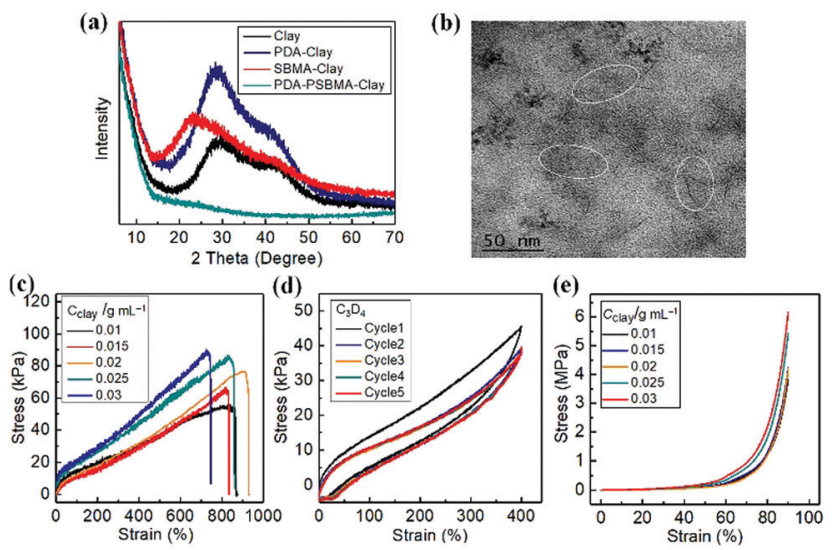

Fig. 2 (a) WAXD profiles of clay, DPA-clay, SBMA-clay, and PDAPSBMA-clay samples. (b) TEM image of the PDA-clay-PSBMA hydrogel. (c) Representative tensile stress-strain curves of hydrogels with different clay concentrations. (d) Cyclic tensile stress-strain curves of the PDAclay-PSBMA hydrogel (DA $=4 \times 10^{-3} \mathrm{~mol} \mathrm{~L}^{-1}$, clay $=0.02 \mathrm{~g} \mathrm{~mL}^{-1}$ ). (e) The compressive stress-strain curves of the hydrogel with different clay concentrations.

The ionic interactions between the zwitterions and clay platelets accounted for the outstanding mechanical properties of the hydrogels. The addition of dopamine slightly deteriorated the tensile and compressive strength of the hydrogels (Fig. S2a and b, ESI $\dagger$ ). With $0.02 \mathrm{~g} \mathrm{~mL}^{-1}$ clay and $4 \times 10^{-3} \mathrm{~mol} \mathrm{~L}^{-1}$ dopamine, the $\mathrm{C}_{3} \mathrm{D}_{4}$ PDA-clay-PSBMA hydrogel showed a tensile strength of $77 \mathrm{kPa}$ and a compressive strength of 4.2 MPa. Hydrogels with this formulation were used for subsequent studies unless otherwise specified.

\section{Self-healing}

The PDA-clay-PSBMA hydrogels can self-heal under ambient conditions. When the hydrogel was cut and contacted at the fresh surface for 10 hours at $25{ }^{\circ} \mathrm{C}$, the interface was obviously blurred (Fig. 3a). The self-healed hydrogel could be stretched to $500 \%$ strain without breaking (Fig. 3b). After $2 \mathrm{~h}$ healing, the

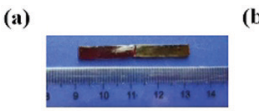

(b)
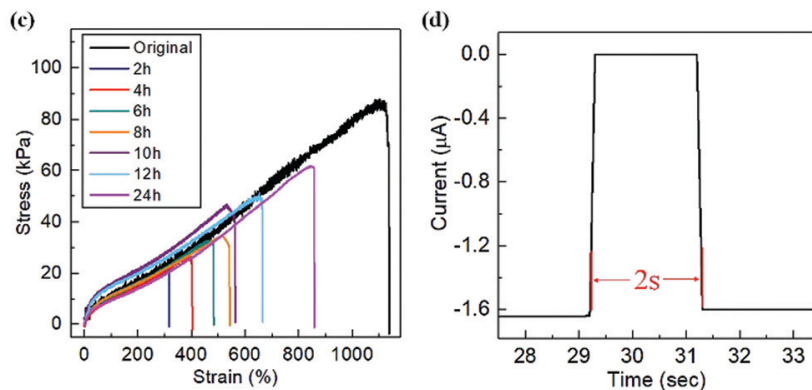

Fig. 3 (a) PDA-clay-PSBMA hydrogels contacted at the freshly cut surface self-healed for $10 \mathrm{~h}$ at room temperature to sustain high stretching (b). (c) Representative tensile stress-strain curves of the original and self-healed hydrogels at room temperature. (d) Self-healing in the electrical property of the freshly cut PDA-clay-PSBMA hydrogel. 
hydrogels could be stretched to $318 \%$. After $24 \mathrm{~h}$, the healed hydrogels could be stretched to $859 \%$ with a fracture strength of $61.5 \mathrm{kPa}$, or an $80 \%$ healing efficiency in fracture strength (Fig. 3c). Moreover, the hydrogels showed much faster healing in the electrical properties. When the hydrogel in a circuit was cut, the current immediately dropped to $0 \mathrm{~A}$, and then it recovered to $97.6 \%$ of the original level in $2 \mathrm{~s}$ after the cut surfaces were placed together (Fig. 3d). The self-healing of the PDA-clay-PSBMA hydrogels favours the environmental adaptability and stability of implantable devices based on the hydrogels for long-lasting sensing. ${ }^{45}$

\section{Tissue adhesion}

The PDA-clay-PAM hydrogels showed robust adhesion to a variety of hydrophobic and hydrophilic surfaces. For example, two glass slides adhered by the hydrogel $(20 \mathrm{~mm} \times 20 \mathrm{~mm} \times$ $2 \mathrm{~mm}$ ) hang a load of $1.5 \mathrm{~kg}$ in air. This assembly could even withstand a load of $1 \mathrm{~kg}$ after soaking in water for $10 \mathrm{~h}$. This phenomenon demonstrates excellent water-proof adhesion, which is critical for adhesion on biotissues. The adhesion performance of the hydrogel to diverse materials was characterized by using lap shear tests on assemblies with the PDAclay-PSBMA hydrogel sandwiched between a pair of glass slides, pig skins, and copper or polytetrafluoroethylene (PTFE) sheets (adhesion area: $20 \times 20 \mathrm{~mm}^{2}$ ). The assemblies were stretched from both ends until separation (adhesion failure) or fracture (cohesion failure), as shown in Fig. 4b. Fig. 4c shows representative adhesion strength-displacement curves for the PDA-clay-PSBMA hydrogels on glass, copper, PTFE and pig skin. The maximum strength is taken as the interface adhesion strength for the interface failure. The adhesion strengths of the hydrogel on glass, $\mathrm{Cu}$, PTFE and pig skin were 94.3, 54, 17.1, and $22.3 \mathrm{kPa}$. Detailed investigations on the effect of clay, PDA, and zwitterionic moieties on the adhesion performance (Fig. S4a-g, ESI $\dagger$ ) suggest that the covalent and non-covalent interactions between polydopamine and the groups on the substrates determine the adhesion of the hydrogels on the substrates from hydrophilic to hydrophobic, and from apolar to polar. Here we mainly focus on the effects of the clay, PDA, and SBMA concentrations on the adhesion performance of the hydrogels on pig skin and some organs.

The interaction of zwitterionic chains with the surface plays critical roles in the adhesion. For example, with constant clay and dopamine contents, the adhesion strength on pig skin monotonically increases (3.1 to $22.3 \mathrm{kPa}$ ) with the zwitterionic monomer content in the hydrogels (Fig. 4d). The adhesion strength could be further enhanced by improving the zwitterionic monomer content in the gels and the dipole-dipole interaction between the hydrogel surface and the polar groups on the tissue surface increases (Fig. 1).

The clay content plays critical roles in the adhesion strength of the hydrogels on pig skin. Essentially, the clay nanosheets provided limited spaces for the oxidation of dopamine. When the clay concentration is low, the cohesive energy of the hydrogel is low, and the mode of adhesion failure at this time is mainly the destruction of the cohesive energy. ${ }^{46}$ As the clay content increases, the cohesive energy of the hydrogel gradually increases, which provides an adhesion strength from 13.4 to $22.3 \mathrm{kPa}$ (Fig. S4d, ESI $\dagger$ ). These values are close to that of the commercial fibrin glue. Nonetheless, as the content of clay was (a)

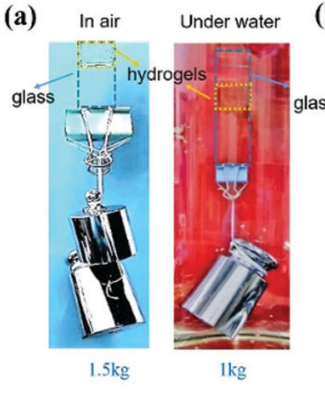

(e)

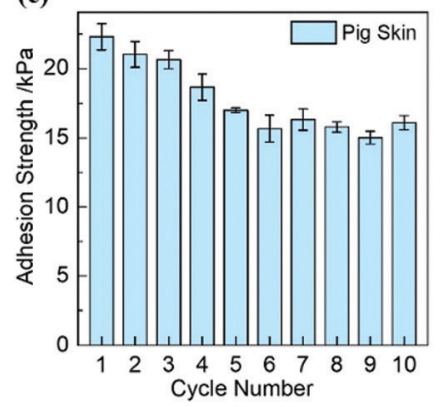

(c)

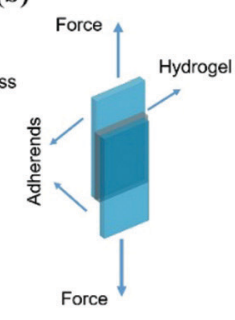

(f)
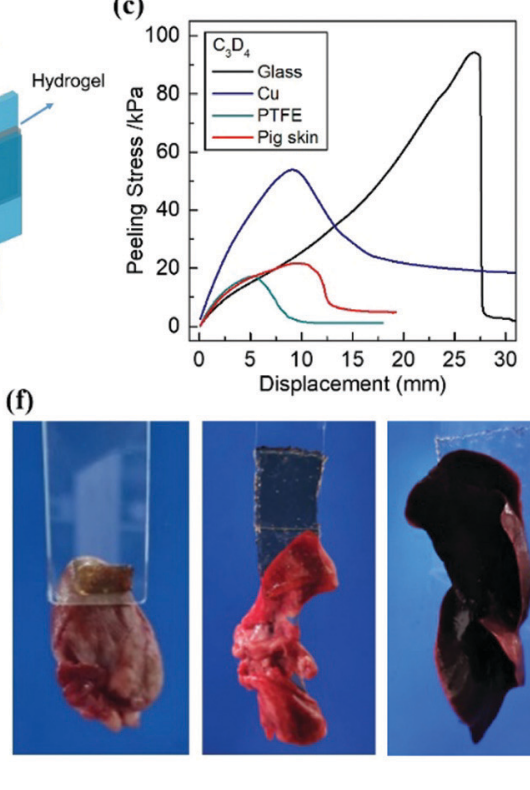

(d)

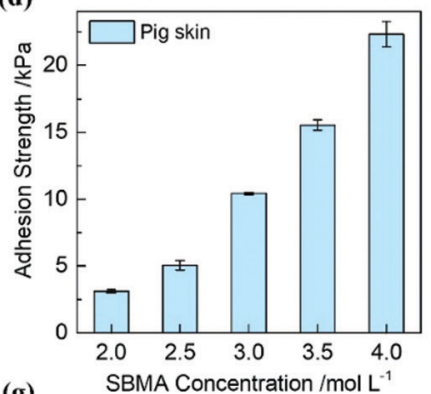

(g)
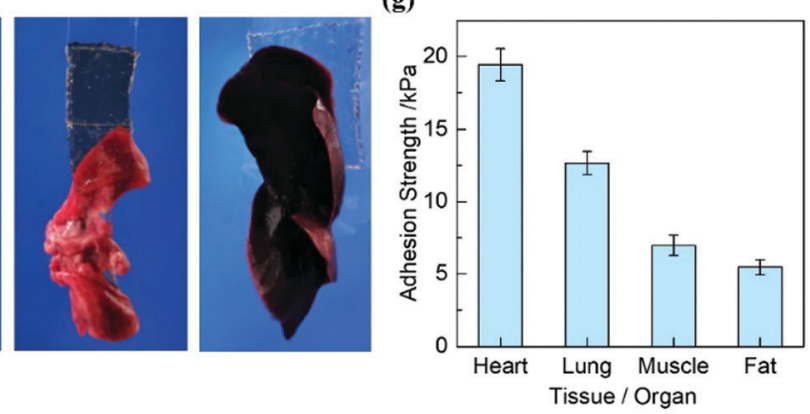

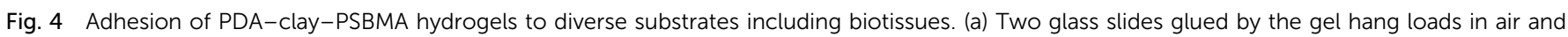

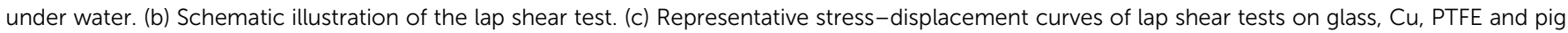

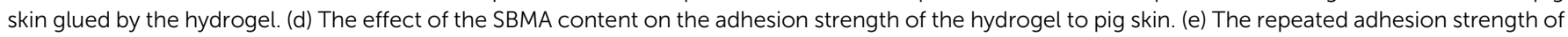
the hydrogels to pig skin. (f) Photographs of hydrogels adhered to rabbit heart, lung, and liver, and (g) the corresponding adhesion strength values. 
raised to $0.03 \mathrm{~g} \mathrm{~mL}^{-1}$, the high clay content may lead to high oxidation of PDA, resulting in a reduction of the free phenolic hydroxyl content and a high cross-linking density, which hinders the mobility of polymer chains at the interface. ${ }^{13}$ Thus, the hydrogel adhesion strength dropped to $9.4 \mathrm{kPa}$.

On the other hand, when the clay content and SBMA monomers are constant, the dopamine concentration dominates the adhesion strength of the hydrogels on pig skin (Fig. S4h, ESI $\dagger$ ). It is encouraging that the adhesive strength of the PDA-clay-PSBMA hydrogels on pig skin $(17.1 \mathrm{kPa})$ was a bit higher than that of fibrin glue $(16.5 \mathrm{kPa})$ and could reach $22.3 \mathrm{kPa}$ with optimal formulations. These results indicate the great potential of the hydrogels to form robust adhesion to tissues for implantable applications in vivo.

The outstanding adhesion of the PDA-clay-PSBMA hydrogels is attributed to the synergistic interactions of SBMA and PDA with the substrates. The zwitterionic polymers with cationic quaternary ammonium groups and anionic sulfonate groups exhibit neutral net charge, but have a high dipole moment. The zwitterionic groups can interact with other charged groups or polar groups through ion-dipole and dipole-dipole interactions. ${ }^{26,32,35}$ Besides, PDA is capable of binding on a variety of surfaces through phenolic hydroxyl chemical reactions similar to those of DOPA molecules in adhesion proteins. ${ }^{4-50}$ On biotissue surfaces, the zwitterionic groups on the zwitterionic polymer chains interact with the $-\mathrm{COOH}$ and $-\mathrm{NH}_{2}$ on the tissue surface through dipole-dipole interactions, while PDA can interact with the tissue surface through hydrogen bonding, Schiff base reaction, or Michael addition (Fig. 1b). To further figure out the importance of electrostatic attraction to the robust adhesion, we introduced $\mathrm{NaCl}$ into the hydrogel to shield the electrostatic interactions between SBMA and the tissue surface. As a result, the adhesion strength of the PDA-clay-PSBMA hydrogel to pig skin is significantly reduced by 55\% (Fig. S4i, ESI $\dagger$ ). The robust adhesion sustained cyclic peeling and re-adhesion for many times. For example, as the hydrogel was adhered onto pig skin and peeled off ten times, the adhesive strength right after each test remained 16.1 kPa (Fig. 4e).

The synergistic effect of PDA and SBMA enables strong adhesion to organs like the liver, heart, and lungs (Fig. 4f). Lap shear tests on tissue-hydrogel assemblies show peeling strength values up to $19.4 \mathrm{kPa}$ (Fig. $4 \mathrm{~g}$ ). In fact, the hydrogels form strong adhesion to the tissues in seconds, and the robust adhesion could survive vigorous shaking (Video S1, ESI $\dagger$ ), which is critical for homeostasis of the heart and pneumothorax. The adhesion is stable against cyclic motions of the beating heart or breathing lung. We simulate heart beating and lung breathing by cyclically pumping air into the heart and lung in vitro. The hydrogel adhered to the heart and lung surfaces deformed conformably without failing or delaminating (Videos S2 and S3, $\mathrm{ESI} \dagger)$. The hydrogel adhesion to biotissues was even waterproof, as demonstrated by immersing the hydrogel-lung assembly in a PBS solution with $\mathrm{pH} 7.2$ and pumping air into the lung to simulate respiration. The hydrogel adhered on the lung sealed the alveoli and prevented bubbling in water (Video S4, ESI $\dagger$ ), suggesting a potential application of the hydrogels as biotissue sealants. The corresponding deformation of the hydrogel occurred with the expansion and contraction of the lung, and it did not fail (Videos S3 and S4, ESI $\dagger$ ).

\section{Conductivity and strain sensitivity}

The zwitterionic PDA-clay-PSBMA hydrogels are ion conductive and sensitive to deformations. Zwitterionic polymer chains serve as a network to assist ion transport. The impedance of the PDA-clay-PSBMA hydrogel was measured by using electrochemical impedance spectroscopy (EIS) (Fig. 5a and Fig. S5a, $\mathrm{ESI} \dagger$ ). The ions released from the exfoliated clay nanosheets give conductivity to the PDA-clay-PSBMA hydrogels, ${ }^{32,51}$ which effectively minimizes the ion pair formation. ${ }^{52}$ The cationic charges on the surface of clay nanosheets may hamper the mobility of anions and thus favour the mobility of cations in polyelectrolytes, ${ }^{53}$ which may promote ion conduction. ${ }^{54}$ The free ions in the hydrogel network increased with the clay content. As the clay content was increased from 0.01 to $0.03 \mathrm{~g} \mathrm{~mL}^{-1}$, the conductivity of the hydrogel increased from 0.0157 to $0.0215 \mathrm{~S} \mathrm{~m}^{-1}$ (Fig. 5b). Besides, as the concentration of dopamine increased, the conductivity showed a maximum of $0.020 \mathrm{~S} \mathrm{~m}^{-1}$ at a clay concentration of $0.04 \mathrm{~g} \mathrm{~mL}^{-1}$ (Fig. S5b, ESI $\dagger$ ).

The hydrogels show a high strain sensitivity. The PDA-clayPSBMA hydrogels were connected to a circuit comprised of a power supply $(2.5 \mathrm{~V})$ and an LED bulb. As the hydrogel was stretched, the LED bulb gradually dimmed. When the hydrogels were gradually released, the resistance almost returned to the initial value, and the bulb gradually became brighter (Fig. S5h, ESI $\dagger$ ). The resistance change ratio during loading is defined as $\left|R-R_{0}\right| / R_{0}$, where $R_{0}$ and $R$ are the initial resistance and that under strain. The strain sensitivity or gauge factor (GF) is defined as GF $=\delta\left[\left|R-R_{0}\right| / R_{0}\right] / \delta \varepsilon$, where $\varepsilon$ is the strain. As the dopamine concentration increased, the resistance change ratio and strain sensitivity of the hydrogel sensor showed a maximum at a clay concentration of $0.04 \mathrm{~g} \mathrm{~mL}^{-1}$ (Fig. S5c and d, $\mathrm{ESI} \dagger$ ). In the strain range of $0-200 \%$, the resistance change ratio and strain sensitivity of the hydrogel sensor are insensitive to the clay content (Fig. $5 \mathrm{c}$ and d). At tensile strain $\geq 200 \%$, the resistance change ratio and sensitivity increased with the clay content (Fig. 5c and d). Besides, under the same strain, the resistance change ratio and sensitivity decreased as the clay content increased (Fig. $5 \mathrm{c}$ and d). The GF of the $\mathrm{C}_{3} \mathrm{D}_{4}$ hydrogel strain sensor was only 1 in the strain range $0-80 \%$, but increased to 2.2 at $300 \%$ strain, which was higher than that of ionic gels reported in the literature $\left(1.5\right.$ at $\left.0-300 \% \operatorname{strain}^{35}\right)$. These hydrogels may provide a large working range for practical applications, which has been difficult for conductive sensors based on elastomers.

Excellent electrical conductivity, sensitivity and adhesion to a variety of material surfaces are critical for the PDA-clayPSBMA hydrogels as sensors for real-time monitoring of human activities. When a hydrogel sensor was mounted on the finger of a volunteer, finger bending to $15^{\circ}, 35^{\circ}$ and $75^{\circ}$ stretched the gel and generated $\left|R-R_{0}\right| / R_{0}$ of $8 \%, 17 \%$ and $34 \%$ (Fig. $5 \mathrm{e}$ ). The resistance change ratio also gradually and linearly increased with the bending angle (Fig. S5i, ESI $\dagger$ ). Such movement monitoring 
(a)

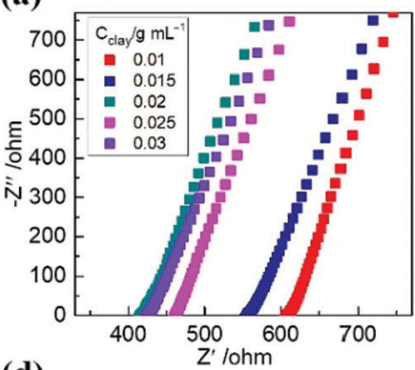

(d)

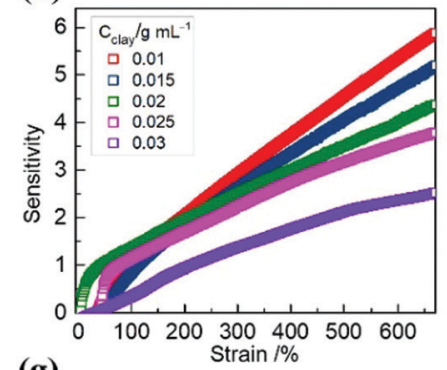

(g)

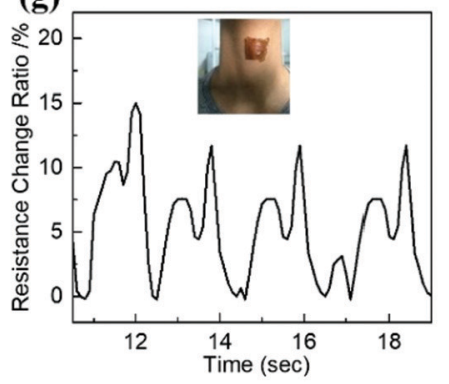

(b)

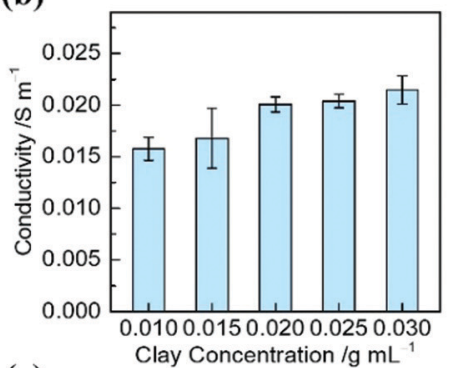

(e)

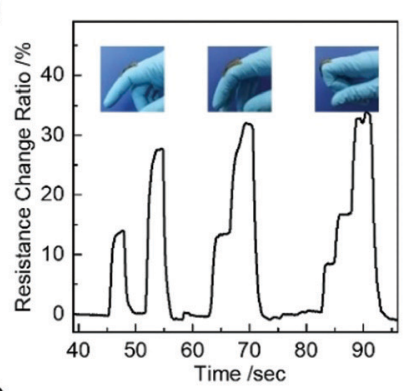

(h)

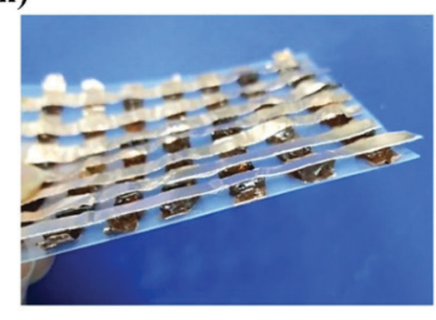

(c)

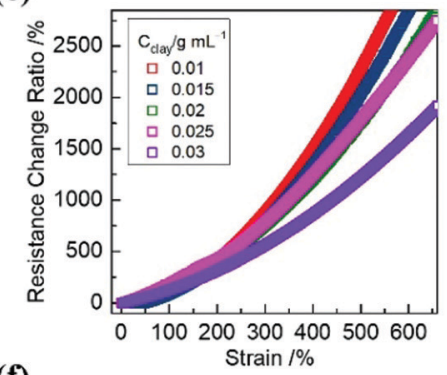

(f)

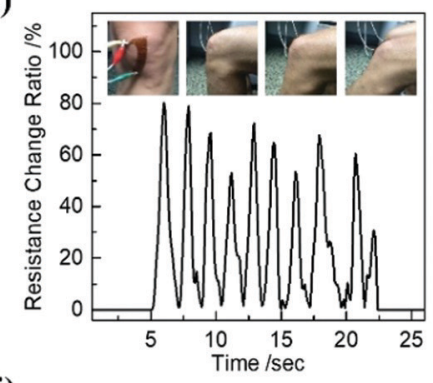

(i)

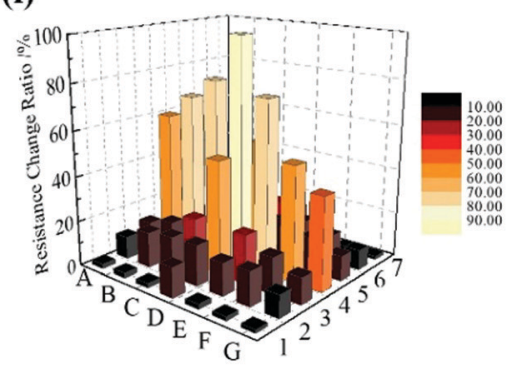

Fig. 5 The effect of the clay content on the (a) impedance, (b) conductivity, (c) resistance change ratio, and (d) strain sensitivity of the hydrogel with $4 \times 10^{-3} \mathrm{~mol} \mathrm{~L}^{-1}$ dopamine. The hydrogels serve as sensors to monitor the repeated bending of the (e) finger and (f) knee of a volunteer. (g) The hydrogel adhered to the throat of a volunteer records swallowing. The hydrogels were fabricated into a $7 \times 7$ array $(\mathrm{h})$ that produces a 3D histogram of pressure on D4 with excellent lateral resolution (i).

is applicable for other joints such as the knee (Fig. 5f). It is noteworthy that the hydrogel sensor adhered firmly to the joint, and sustained vigorous movements as the volunteer quickly jumped rope (Video S5, ESI $\dagger$ ). Furthermore, the hydrogel sensor was able to detect tiny human motions. The strain sensor attached onto the throat sensitively responded to subtle muscle movements to present the real-time resistance change ratio when the volunteer swallowed (Fig. 5g). These results demonstrate great potential of adhesive conductive hydrogel sensors for wearable devices.

The ion conductive hydrogels have been fabricated into sensor arrays to detect 2D distributions of pressure or strain, which is the basis of many wearable or implantable sensory devices. A prototype $7 \times 7$ array of hydrogels connected by copper tape was fabricated, with each hydrogel cube $(5 \mathrm{~mm} \times$ $5 \mathrm{~mm} \times 2 \mathrm{~mm}$ ) acting as an independent sensor unit/pixel (Fig. $5 \mathrm{~h}$ ). When a $100 \mathrm{~g}$ weight ( $22 \mathrm{~mm}$ bottom diameter) was placed at D4, for example, it covered 7/10 of four sensor pixels neighbouring D4. Thus, the pressure rendered changes in resistance for pixels underneath the weight. The resistance change ratio at D4 was higher than $90 \%$, while the resistance change ratio of the neighbouring sensor pixels was between
$50 \%$ and $70 \%$ (Fig. 5i). In contrast, no significant signals were observed for other sensor pixels. No crosstalk occurred. This array can be extended to make highly integrated arrays of very small gel pixels for E-skin.

Real-time wireless monitoring of organ motions by using ion conductive and adhesive hydrogel sensors

The above results strongly suggest promise of the tissue-adhesive and conductive PDA-clay-PSBMA hydrogels as sensors to monitor the motions of highly dynamic organs. First, the strong adhesion of the hydrogel to organs enabled rapid sealing of wounds in the lungs, liver or heart. Meanwhile, the ability to detect subtle stress and strain enables in situ monitoring of the movement of the lungs in real time. Here, we demonstrate wireless transmission of the collected signals from simulated dynamic lung and heart motions to the computer for diagnosis. To simulate pneumothorax, a rabbit lung was punctured with a needle. Then a $(30 \mathrm{~mm} \times 10 \mathrm{~mm} \times 2 \mathrm{~mm})$ hydrogel patch was adhered to the wound. Lung breathing was simulated in vitro by pumping air into the lung. The gel patch adhered to the damaged lung and completely sealed the wound. No bubbling took place as air was pumped into the lung under 
(a)

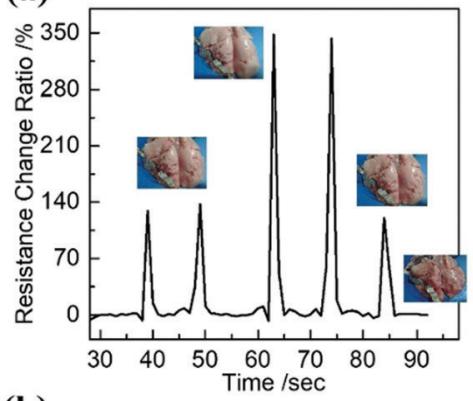

(b)

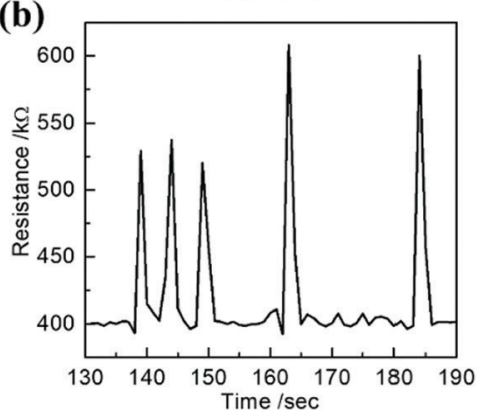

(c)

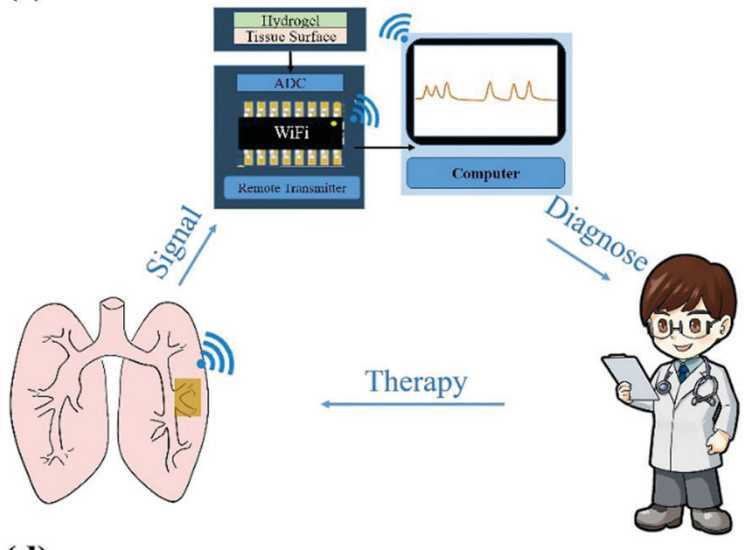

(d)
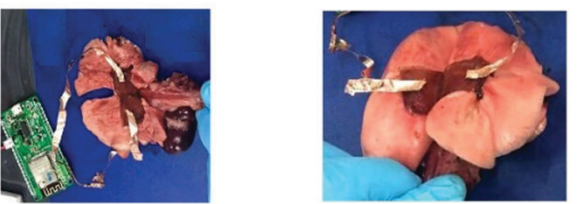

Fig. 6 Real time monitoring and wireless transmission of simulated lung breathing by hydrogel sensors. (a) Wired transmission of the resistance change ratio and (b) wireless transmission of the resistance over time generated from simulated lung breathing. (c) Schematic illustration showing the wireless monitoring of lung breathing. (d) Photos of hydrogel sensors mounted on a rabbit lung with wired (left) and wireless (right) transmission.

water (Video S4, ESI $\dagger$ ). Meanwhile, each time the air was pumped into the lung, the hydrogel was stretched to generate a resistance change ratio of $140 \%$ or $350 \%$ (Fig. $6 a$ ), depending on the expansion ratio of the lung. As the lung relaxed, the resistance change ratio almost returned to the initial value (Fig. 6a). This procedure could be repeated many times without failure of the adhesion. The robust adhesion and sealing of the lung allowed for normal breathing, which is highly significant for clinical applications to treat pneumothorax and for subsequent monitoring of the status of the lung (Fig. 6c).

For post-treatment monitoring, wireless transmission is very important for implantable sensors. Thanks to the strong adhesion, the conductive hydrogel sensor could be directly adhered on the lungs or heart, without suturing or gluing with chemicals (Fig. 6d), to monitor human activity by coupling wireless transmitters and wirelessly communicating with computers (Fig. 6c). We compared the output signal quality obtained from wireless transmission and conventional wired transmission. Fig. 6b shows the real-time sensing performance of the PDAclay-PSBMA hydrogel-based sensor adhered onto the lung with different breathing. The signal output through wireless transmission is recorded as resistance since it was converted and restored from the wireless data. The larger expansion of the lung led to greater stretching of the sensor, which produced electrical signals that were wirelessly transmitted to and processed by a computer. The relative peak shape and height ratio were consistent with those directly obtained through wired monitoring (Fig. 6a) when the air was blown into the lung. It is noted that the signals collected by wireless and wired transmission were not significantly different in terms of sensitivity. The hydrogel sensor was able to accurately monitor and quantify real-time strain changes through wireless transmission (Video S6, ESI $\dagger$ ). Thus, the results could help physicians and medical professionals to perform remote activity monitoring to help patients. Moreover, such in situ monitoring and wireless transmission could be combined with intelligent terminal and artificial intelligence (AI) to achieve remote diagnostics and medication of patients and people needing help (Fig. 6c).

Biocompatibility is critical for implantable devices. To assess the biocompatibility in vivo, PDA-clay-PSBMA hydrogels were implanted under the skin of mice to examine the immune response (Fig. 7a). At specific times, the hydrogels along with surrounding tissues were collected, microtomed, and analysed using H\&E staining (Fig. 7b). On the first day of implantation, many cells, including multinucleated cells, were found in the tissue near the hydrogels, indicating inflammatory responses against the hydrogel as a foreign body. On the fifth day, the inflammatory cell density was greatly reduced. On the 7 th day, the cell density returned to normal, no polynuclear cells were aggregated, and the inflammatory reaction disappeared. These results indicate that the hydrogels did not cause immunological rejection in mice. In order to further test the biological safety of the hydrogel, during the subcutaneous implantation experiments, routine blood tests were conducted on the mice. The results of two-week implantation showed no indications of toxicity (Table S1, ESI $\dagger$ ). These results suggest that the PDAclay-PSBMA hydrogels had excellent biocompatibility. All the results of the hydrogels shown and discussed above lay the foundation for applications as implantable devices to monitor human health.

The nano-composite hydrogels have excellent adhesion properties on biotissues through the synergistic effect of zwitterions 

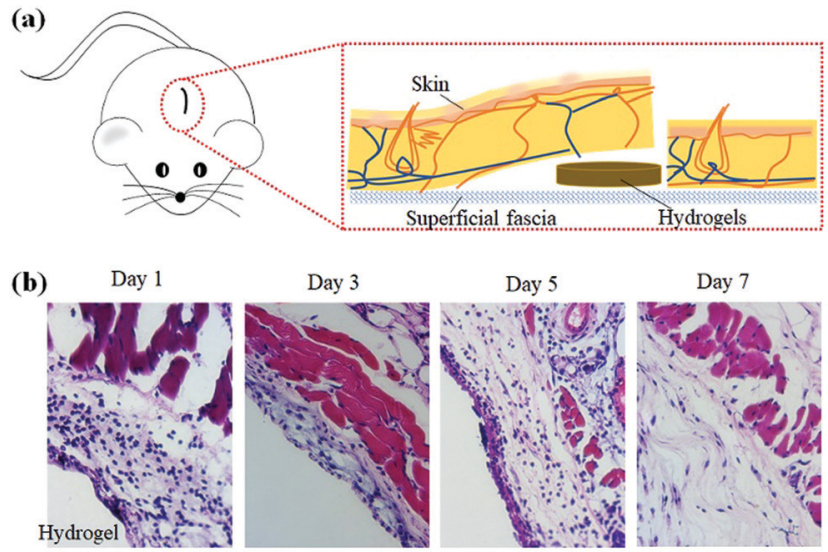

Fig. 7 In vivo biocompatibility evaluation of PDA-clay-PSBMA. (a) Schematics of a mouse model for subcutaneous implantation of hydrogels. (b) H\&E staining of skin tissues surrounding the hydrogels after implantation for $1,3,5$, and 7 days.

and dopamine. Mussel-inspired adhesives are able to adhere to various substrates through powerful catechol chemistry. ${ }^{48-50,55,56}$ However, the dopamine-based adhesion to the skin surface is only $5.2 \mathrm{kPa}^{30}$ For practical applications, robust adhesion and conformal contact between conductive hydrogel sensors and biotissues are needed for efficient electrical signal transmission. Zwitterionic groups provide a high dipole moment that favours excellent adhesion to many surfaces through ion-dipole or dipole-dipole interactions. ${ }^{26,32,35}$ The combination of dopamine and zwitterionic groups significantly improves the adhesion strength to $19.4 \mathrm{kPa}$ to tissues, a little higher than the commercially used fibrin adhesives.

On the other hand, the zwitterionic groups can assist in separating the cationic and anionic counterions during ion migration, and thus give ion conductivity. ${ }^{34}$ The conductivity and resistance change with deformation of the hydrogels. As a result, the PDA-clay-PSBMA hydrogels show high sensitivities at low ( $\mathrm{GF}=1.25,0 \%<$ strain $<80 \%)$ and high strains (GF $=2,0 \%<$ strain $<200 \%$ ). These values are superior to many other ion conductive hydrogels previously reported in the literature. $^{57-59}$

The ion conductive hydrogels, possessing both strong adhesion to tissues and high strain sensitivity, are promising for clinical applications. The robust adhesion to tissues may enable easy fixing of implantable sensors to tissue and organs for in situ monitoring of human health, without using suturing, which is usually used in surgery to fix implantable devices. Stability and reliability are very important for implantable devices. For dynamic organs, excellent strength, toughness, and fatigue resistance are critical for stable and reliable sensors to work in vivo. Here, physical adsorption of polymer chains to clay nanosheets and dipole-dipole association of the zwitterionic chains serve as energy dissipation mechanisms to ensure high mechanical properties and self-healing. The rapid self-healing in electrical performance is beneficial for the reliability of the sensors.

To date, most flexible sensors are employed in conventional electronics for signal transmission and interpretation. For wearable and implantable devices, it will be much more useful if wireless communication technologies are integrated. This is of special significance for in vivo applications. Our results demonstrate the feasibility of using biotissue-adhesive hydrogel sensors for remote monitoring of dynamic motions of organs through wireless transmission.

\section{Conclusions}

In this work, we demonstrate novel tissue-adhesive and conductive PDA-clay-PSBMA hydrogels. Adequate catechol groups in the hydrogel and reversible physical interactions between the zwitterionic and other surfaces provide reproducible robust adhesion to various materials. Most importantly, the hydrogels can adhere on a variety of biological tissue surfaces with adhesion strength up to $19.4 \mathrm{kPa}$ (on the heart). It is worth noting that the hydrogels retained adhesion in wet and even underwater environments. Excellent adhesion and biocompatibility are beneficial to the repair of tissue defects. Moreover, the hydrogel can self-heal without any external stimuli. The outstanding ion conductivity and high sensitivity are beneficial for the PDA-clay-PSBMA hydrogels as flexible and wearable strain or pressure sensors for direct monitoring of large-scale human movements and physiological signals, including swallowing and the bending of the knuckles and knee joints. The direct adhesion of the hydrogels to the lungs was demonstrated to monitor simulated respiratory signals. The results are encouraging for potential applications of such stretchable, tough, and tissue-adhesive materials for implantable and wearable sensors that are designed for monitoring of dynamic organs in vivo. In conclusion, the PDA-clay-PSBMA hydrogels could hold considerable promise for a variety of applications such as tissue adhesion strain sensors, medical electrodes and wearable devices.

\section{Experimental section}

\section{Materials}

A synthetic hectorite "LAPONITE ${ }^{\circledR}$ XLG $^{\circledR}$ ", was provided by Zhejiang Fenghong New Materials Co. Ltd. [2-(Methacryloyloxy)ethyl]dimethyl-(3-sulfopropyl) ammonium hydroxide (SBMA, 97\%), ammonium persulfate (APS), and $N, N^{\prime}$-methylene bis(acrylamide) (MBAA, 99\%) were purchased from Aladdin (Shanghai, China). Dopamine hydrochloride (DA) was purchased from Sigma-Aldrich. All these reagents were used without further purification. Deionized water $\left(18.2 \mathrm{M} \Omega\right.$, at $\left.25{ }^{\circ} \mathrm{C}\right)$ was prepared before use.

\section{Preparation of polydopamine zwitterionic nanocomposite hydrogels}

An aqueous suspension of LAPONITE ${ }^{\circledR}$ XLG was prepared at room temperature. Dopamine was added to the dispersed LAPONITE $^{\circledR}$ XLG solution and stirred for $5 \mathrm{~h}$ to prepare a LAPONITE $^{\circledR}$ XLG/DA suspension, and then SBMA monomers, APS and MBAA were added to the LAPONITE $^{\circledR}$ XLG/DA 
suspension and stirred for $1 \mathrm{~h}$ to produce a homogeneous solution, where the molar ratio of MBAA to SBMA was $3 \times 10^{-4}$. Finally, PDA-clay-PSBMA hydrogels were obtained by free radical polymerization at $60{ }^{\circ} \mathrm{C}$ for $24 \mathrm{~h}$.

\section{Microstructure characterization of the hydrogels}

The hydrogels were freeze-dried and freeze-fractured in liquid nitrogen, and sputtered with platinum on the cross-section for SEM (S4800, Hitachi, Japan) imaging at $4 \mathrm{kV}$.

TEM imaging was performed by using a JEM-2100F transmission electron microscope (JEM-2100F, Japan) at $200 \mathrm{kV}$ on samples cast on an ultra-thin carbon film.

X-ray diffraction (XRD) measurements of clay nanosheets, and PDA-clay, SBMA-clay, PDA-clay-SBMA, PDA-clay-PSBMA and PSBMA-clay composites were conducted by using an X-ray powder diffractometer (D8 ADVANCE, BRUKER, Germany) at $40 \mathrm{kV}$ and $4 \mathrm{~mA}$.

\section{Mechanical testing}

The mechanical properties of the hydrogels were investigated by using a Universal Testing Machine (Instron 5567, USA) at room temperature. Tensile tests were conducted on rectangular specimens $(20 \mathrm{~mm} \times 5 \mathrm{~mm} \times 2 \mathrm{~mm})$ at a crosshead speed of $100 \mathrm{~mm} \mathrm{~min}^{-1}$. Cyclic loading-unloading tensile tests were performed to a strain of $400 \%$. Every cycle was conducted after 3 min of complete recovery of the hydrogel. Compression tests were conducted on cylindrical samples (height $2 \mathrm{~mm}$, diameter $13 \mathrm{~mm})$.

\section{Adhesion measurements}

The adhesion performance of the PDA-clay-PSBMA hydrogels was determined through lap shear tests using a Universal Testing Machine (Instron 5567, USA). A hydrogel $(20 \mathrm{~mm} \times$ $20 \mathrm{~mm} \times 2 \mathrm{~mm}$ ) was sandwiched between two glass plates, copper sheets, PTFE plates, or tissues/organs (pig skin, heart, lung, pork muscle, or fat). All tissues are fresh and the surfaces of the tissues are wiped dry without fluid-like liquids. Pig skin, pork muscle, and fat were purchased from a local Wumart. Heart, lung and liver were retrieved from sacrificed New Zealand white rabbits (2.5-3 kg each). A $100 \mathrm{~g}$ load was applied for $5 \mathrm{~min}$ to form good contact before testing. Repeated lap shear tests were also conducted on samples after cyclic peeling off and re-adhesion for $5 \mathrm{~min}$. All tests were performed at a crosshead speed of $100 \mathrm{~mm} \mathrm{~min} \mathrm{~min}^{-1}$ at room temperature. Measurements were repeated at least five times to report results as mean \pm standard deviation.

\section{Electrical property measurements}

The electrochemical impedance measurements of the PDAclay-PSBMA hydrogels were performed by using a Solartron 1470 E multi-channel potentiostat electrochemical workstation (Solartron Public Corp., Ltd). The impedance data were analysed by using the software ZView according to the Randles equivalent circuit. The resistance changes were obtained by using an electrochemical analyser/workstation (CHI600E, China). The gauge factor is defined as GF $=\delta\left[\left|R-R_{0}\right| / R_{0}\right] / \delta \varepsilon=\delta\left(\Delta R / R_{0}\right) / \delta \varepsilon$, where $R_{0}$ and $R$ are the resistances of the original and stretched hydrogels, and $\varepsilon$ is the strain of the hydrogel.

\section{Adhesive strain sensors to monitor organ motions and wireless transmission}

An electrochemical analyser/workstation (CHI600E, China) was used to monitor the electrical signal changes of hydrogels adhered to bending joints and organs. The finger, knee, and throat movement/vibration monitoring experiments were performed on a volunteer, with informed consent obtained from the volunteer. Wireless transmission equipment was mounted onto the gel sensor for remote monitoring of the electrical signal changes of hydrogel sensors adhered to a lung and heart with simulated motions in vitro. The heart and lung were retrieved from sacrificed New Zealand white rabbits (2.5-3 kg each).

\section{In vivo immune response evaluation}

Hydrogels were embedded under the skin of the back of mice to evaluate the immune response and biocompatibility of the hydrogels. Before implantation, hydrogel discs $(5 \mathrm{~mm}$ in diameter and $2 \mathrm{~mm}$ in thickness) were sterilized with $70 \%$ ethanol for $24 \mathrm{~h}$ and soaked in PBS to remove residue ethanol. Briefly, seven male mice (ICR mouse) weighing 28-30 g were used. After being anesthetized with pentobarbital ( $2 \mathrm{wt} \%$, $2 \mathrm{~mL} \mathrm{~kg}^{-1}$ ), the dorsal area of the mice was totally depilated. PDA-clay-PSBMA hydrogels were implanted into three tissue pouches on the back of each mouse, and then the wounds were closed by using a Tegaderm TM dressing (3M, St. Paul, MN, USA). The mice were observed daily and euthanized on the first, third, fifth and seventh days after implantation. The samples were retrieved, embedded in paraffin and sliced, followed by staining using haematoxylin eosin for optical observations. Moreover, the blood of mice with hydrogels implanted for two weeks was collected for routine blood testing. All experimental mice were housed in the Ningbo University Animal Service Centre. All the animal experiments were performed in compliance with the guidelines for the Care and Use of Research Animals established by the Institutional Animal Ethical Committee (IAEC) of Ningbo University. All surgical procedures were approved by the IAEC of Ningbo University, Ningbo, China.

\section{Conflicts of interest}

There are no conflicts to declare.

\section{Acknowledgements}

This study was supported by the National Natural Science Foundation of China (51873224) and the National Key Research and Development Program of China (2016YFC1101902).

\section{Notes and references}

1 H. C. Koydemir and A. Ozcan, Annu. Rev. Analyt. Chem., 2018, 11, 127-146. 
2 C.-H. Lai, D. S. Ashby, N. H. Bashian, J. Schoiber, T.-C. Liu, G. S. Lee, S.-Y. Chen, P.-W. Wu, B. C. Melot and B. S. Dunn, Adv. Energy Mater., 2019, 9, 1900226.

3 B. W. Walker, R. Portillo Lara, E. Mogadam, C. Hsiang Yu, W. Kimball and N. Annabi, Prog. Polym. Sci., 2019, 92, 135-157.

4 Y. Zhang, J. Ding, B. Qi, W. Tao, J. Wang, C. Zhao, H. Peng and J. Shi, Adv. Funct. Mater., 2019, 29, 1902834.

5 L. Guo, Z. Chen, D. Zhang, J. Liu and J. Pan, IEEE Int. Things J., 2019, 6, 9633-9644.

6 S. Kim, R. Bhandari, M. Klein, S. Negi, L. Rieth, P. Tathireddy, M. Toepper, H. Oppermann and F. Solzbacher, Biomed. Microdevices, 2009, 11, 453-466.

7 I. R. Stephen and V. S. Krishna, Neurosurg. Focus, 2009, 27, E5.

8 D. Khodagholy, J. N. Gelinas, T. Thesen, W. Doyle, O. Devinsky, G. G. Malliaras and G. Buzsáki, Nat. Neurosci., 2015, 18, 310-315.

9 J. Sedó, J. Saiz-Poseu, F. Busqué and D. Ruiz-Molina, Adv. Mater., 2013, 25, 653-701.

10 E. Faure, C. Falentin-Daudré, C. Jérôme, J. Lyskawa, D. Fournier, P. Woisel and C. Detrembleur, Prog. Polym. Sci., 2013, 38, 236-270.

11 B. P. Lee, P. B. Messersmith, J. N. Israelachvili and J. H. Waite, Annu. Rev. Mater. Res., 2011, 41, 99-132.

12 M. J. Brennan, B. F. Kilbride, J. J. Wilker and J. C. Liu, Biomaterials, 2017, 124, 116-125.

13 L. Han, X. Lu, K. Liu, K. Wang, L. Fang, L.-T. Weng, H. Zhang, Y. Tang, F. Ren, C. Zhao, G. Sun, R. Liang and Z. Li, ACS Nano, 2017, 11, 2561-2574.

14 G. Ge, W. Yuan, W. Zhao, Y. Lu, Y. Zhang, W. Wang, P. Chen, W. Huang, W. Si and X. Dong, J. Mater. Chem. A, 2019, 7, 5949-5956.

15 T. Jia, Y. Wang, Y. Dou, Y. Li, M. Jung de Andrade, R. Wang, S. Fang, J. Li, Z. Yu, R. Qiao, Z. Liu, Y. Cheng, Y. Su, M. Minary-Jolandan, R. H. Baughman, D. Qian and Z. Liu, Adv. Funct. Mater., 2019, 29, 1808241.

16 Y. Liu, K. He, G. Chen, W. R. Leow and X. Chen, Chem. Rev., 2017, 117, 12893-12941.

17 X. Shi, S. Liu, Y. Sun, J. Liang and Y. Chen, Adv. Funct. Mater., 2018, 28, 1800850.

18 Y. Zhou, C. Wan, Y. Yang, H. Yang, S. Wang, Z. Dai, K. Ji, H. Jiang, X. Chen and Y. Long, Adv. Funct. Mater., 2019, 29, 1806220.

19 X. Liang, T. Zhao, P. Zhu, Y. Hu, R. Sun and C.-P. Wong, ACS Appl. Mater. Interfaces, 2017, 9, 40857-40867.

20 L.-Q. Tao, K.-N. Zhang, H. Tian, Y. Liu, D.-Y. Wang, Y.-Q. Chen, Y. Yang and T.-L. Ren, ACS Nano, 2017, 11, 8790-8795.

21 J. Oh, J. C. Yang, J.-O. Kim, H. Park, S. Y. Kwon, S. Lee, J. Y. Sim, H. W. Oh, J. Kim and S. Park, ACS Nano, 2018, 12, 7546-7553.

22 Z. Wang, J. Chen, Y. Cong, H. Zhang, T. Xu, L. Nie and J. Fu, Chem. Mater., 2018, 30, 8062-8069.

23 M. Jung, K. Kim, B. Kim, K.-J. Lee, J.-W. Kang and S. Jeon, Nanoscale, 2017, 9, 17212-17219.
24 C. Yang and Z. Suo, Nat. Rev. Mater., 2018, 3, 125-142.

25 J.-Y. Sun, C. Keplinger, G. M. Whitesides and Z. Suo, Adv. Mater., 2014, 26, 7608-7614.

26 B. Yang and W. Yuan, ACS Appl. Mater. Interfaces, 2019, 11, 40620-40628.

27 X. Zhang, N. Sheng, L. Wang, Y. Tan, C. Liu, Y. Xia, Z. Nie and K. Sui, Mater. Horiz., 2019, 6, 326-333.

28 W. Kong, C. Wang, C. Jia, Y. Kuang, G. Pastel, C. Chen, G. Chen, S. He, H. Huang, J. Zhang, S. Wang and L. Hu, Adv. Mater., 2018, 30, 1801934.

29 B. Ryplida, K. D. Lee, I. In and S. Y. Park, Adv. Funct. Mater., 2019, 29, 1903209.

30 M. Liao, P. Wan, J. Wen, M. Gong, X. Wu, Y. Wang, R. Shi and L. Zhang, Adv. Funct. Mater., 2017, 27, 1703852.

31 Q. Zhang, X. Liu, L. Duan and G. Gao, Chem. Eng. J., 2019, 365, 10-19.

32 L. Wang, G. Gao, Y. Zhou, T. Xu, J. Chen, R. Wang, R. Zhang and J. Fu, ACS Appl. Mater. Interfaces, 2019, 11, 3506-3515.

33 C. K. Roy, H. L. Guo, T. L. Sun, A. B. Ihsan, T. Kurokawa, M. Takahata, T. Nonoyama, T. Nakajima and J. P. Gong, Adv. Mater., 2015, 27, 7344-7348.

34 X. Peng, H. Liu, Q. Yin, J. Wu, P. Chen, G. Zhang, G. Liu, C. Wu and Y. Xie, Nat. Commun., 2016, 7, 11782.

35 Z. Wang, J. Chen, L. Wang, G. Gao, Y. Zhou, R. Wang, T. Xu, J. Yin and J. Fu, J. Mater. Chem. B, 2019, 7, 24-29.

36 Y. Bu, L. Zhang, G. Sun, F. Sun, J. Liu, F. Yang, P. Tang and D. Wu, Adv. Mater., 2019, 31, 1901580.

37 S. Hong, Y. S. Na, S. Choi, I. T. Song, W. Y. Kim and H. Lee, Adv. Funct. Mater., 2012, 22, 4711-4717.

38 T. Bai, S. Liu, F. Sun, A. Sinclair, L. Zhang, Q. Shao and S. Jiang, Biomaterials, 2014, 35, 3926-3933.

39 M. Kobayashi and A. Takahara, Polym. Chem., 2013, 4, 4987-4992.

40 H. G. Silverman and F. F. Roberto, Mar. Biotechnol., 2007, 9, 661-681.

41 B. Chen, J. R. G. Evans, H. C. Greenwell, P. Boulet, P. V. Coveney, A. A. Bowden and A. Whiting, Chem. Soc. Rev., 2008, 37, 568-594.

42 Z. Xu, Sci. Rep., 2013, 3, 2914.

43 Z. Ma, H. Liu and B. Wu, Br. J. Clin. Pharmacol., 2014, 77, 410-420.

44 J. Ning, G. Li and K. Haraguchi, Macromolecules, 2013, 46, 5317-5328.

45 X. Pan, Q. Wang, P. He, K. Liu, Y. Ni, L. Chen, X. Ouyang, L. Huang, H. Wang and S. Xu, Chem. Eng. J., 2020, 379, 122271.

46 H. Yuk, T. Zhang, S. Lin, G. A. Parada and X. Zhao, Nat. Mater., 2016, 15, 190-196.

47 S. Ma, Y. Wu and F. Zhou, Curr. Opin. Colloid Interface Sci., 2020, 47, 84-98.

48 H. Lee, S. M. Dellatore, W. M. Miller and P. B. Messersmith, Science, 2007, 318, 426.

49 W. Zhang, R. Wang, Z. Sun, X. Zhu, Q. Zhao, T. Zhang, A. Cholewinski, F. Yang, B. Zhao, R. Pinnaratip, P. K. Forooshani and B. P. Lee, Chem. Soc. Rev., 2020, 49, 433-464. 
50 Q. Guo, J. Chen, J. Wang, H. Zeng and J. Yu, Nanoscale, 2020, 12, 1307-1324.

51 P. Wei, T. Chen, G. Chen, H. Liu, I. T. Mugaanire, K. Hou and M. Zhu, ACS Appl. Mater. Interfaces, 2020, 12, 3068-3079.

52 M. Riley, P. S. Fedkiw and S. A. Khan, J. Electrochem. Soc., 2002, 149, A667.

53 M. J. Koh, H. Y. Hwang, D. J. Kim, H. J. Kim, Y. T. Hong and S. Y. Nam, J. Mater. Sci. Technol., 2010, 26, 633-638.

54 R. Prasanth, N. Shubha, H. H. Hng and M. Srinivasan, Eur. Polym. J., 2013, 49, 307-318.
55 M. Shin, S.-G. Park, B.-C. Oh, K. Kim, S. Jo, M. S. Lee, S. S. Oh, S.-H. Hong, E.-C. Shin, K.-S. Kim, S.-W. Kang and H. Lee, Nat. Mater., 2017, 16, 147-152.

56 R. Wang, J. Li, W. Chen, T. Xu, S. Yun, Z. Xu, Z. Xu, T. Sato, B. Chi and H. Xu, Adv. Funct. Mater., 2017, 27, 1604894.

57 C. Shao, M. Wang, L. Meng, H. Chang, B. Wang, F. Xu, J. Yang and P. Wan, Chem. Mater., 2018, 30, 3110-3121.

58 Y.-J. Liu, W.-T. Cao, M.-G. Ma and P. Wan, ACS Appl. Mater. Interfaces, 2017, 9, 25559-25570.

59 Z. Wang, Y. Cong and J. Fu, J. Mater. Chem. B, 2020, 8, 3437-3459. 